\title{
Solving delay differential equations using modified 2-point block method
}

\begin{abstract}
The performance of a modified 2-point block method for solving delay differential equations (DDE) has been investigated. The proposed method computes the numerical solution at two points simultaneously and produces two new equally spaced solution values within a block. This method is based on a pair of explicit and implicit of Adams formulas which are implemented in PE(CE)s mode. The Newton divided difference interpolation has been used to approximate the delay solutions and the algorithm is implemented in variable step size. Numerical results demonstrate the efficiency of the proposed method when solving delay differential equations.
\end{abstract}

Keyword: Block method; Delay differential equations; Variable step size 